\title{
Public-Health-Aufgabe am Puls der Zeit: Schulärztin/Schularzt
}

\begin{abstract}
Annemarie Tschumpera, Nino Künzli ${ }^{b}$, Beat Gloor ${ }^{c}$, Julia Dratvad
${ }^{a}$ Dr. med., Co-Präsidentin der Vereinigung der Schulärztinnen und Schulärzte der Schweiz, Gesundheitsdienst der Stadt Bern; b Prof. Dr. med. et phil., Direktor der Swiss School of Public Health (SSPH+), Vizedirektor und Leiter Departement Lehre und Ausbildung, Swiss Tropical and Public Health Institute, Basel; ' Facharzt Allgemeine Innere Medizin, Hausarzt und Schularzt, Turbenthal; d PD Dr. med., Präsidentin der Schweizerischen Gesellschaft der Fachärztinnen und -ärzte für Prävention und Gesundheitswesen, Swiss Tropical and Public Health Institute, Basel
\end{abstract}

Schulärztinnen und Schulärzte sind ein wichtiger Pfeiler der öffentlichen Gesundheit in der Schweiz. Mit ihrer vielseitigen Tätigkeit an der Schnittstelle zwischen Gesundheit und Bildung haben sie die Möglichkeit, zu Gesundheits- und Bildungschancen für alle Kinder beizutragen. Um dieses Potential zu nutzen, ist spezifisches Fachwissen erforderlich.

\section{Médecin scolaire - La santé publique, une mission d'aujourd'hui}

Les services de médecine scolaire soutiennent les enfants et les adolescents dans leur évolution scolaire, car en coopération avec les médecins traitants et les écoles ils s'attellent aux problèmes médicaux préjudiciables à la scolarité. Grâce à l'école, les médecins scolaires parviennent à toucher tous les enfants et les adolescents, même ceux appartenant à des groupes cibles généralement difficiles à aborder. Ils ont de plus la possibilité d'observer les tendances collectives en matière de santé, d'en référer aux autorités sanitaires responsables, dans l'école et dans la commune, et d'exiger, en vertu de leurs fonctions, que les mesures nécessaires soient prises. Les conseils et la coopération des médecins scolaires sont également sollicités pour la promotion de la santé à l'école. Le concept "d'école en santé» a un énorme impact sur la politique de la santé. II est donc important que les médecins conservent un rôle majeur dans ce domaine interprofessionnel. Pour ce faire, il faut aussi prévoir des formations continues pour acquérir les connaissances spécifiques à la médecine scolaire.

Bildung und Gesundheit hängen eng zusammen: Der Bildungsabschluss ist eine der wichtigsten Determinanten für die lebenslange Gesundheit. Und umgekehrt sind die gesundheitlichen Lernvoraussetzungen entscheidend für den Bildungserfolg. Die Spezialität der schulärztlichen Tätigkeit ist ihre Verortung an der Schnittstelle zwischen Gesundheit und Bildung und ihre Nähe zur Lebenswelt Schule.

Schulärztinnen und Schulärzte haben die Aufgabe, Kinder auf gesundheitlicher Ebene in der Entfaltung ihres Bildungspotentials zu unterstützen. Sie sehen das einzelne Kind und dessen gesundheitliche Situation in der Schule, sei es bei schulärztlichen ScreeningUntersuchungen oder anlässlich von Untersuchungen und Beratungen in spezifischen Problemlagen (Absentismus, gesundheitliche Fragestellungen im Kontext der Schule, Kindeswohlgefährdung etc.). Sie können Jugendliche, Eltern und Lehrpersonen beraten, Schülerinnen und Schüler zu Abklärungen oder Therapien weiter weisen, für chronisch kranke Kinder den Informationsfluss zwischen behandelnden Ärztinnen und Ärzten, Eltern, Kind und Schule erleichtern oder gemeinsam mit der Schulleitung für notwendige Massnahmen in der Schule sorgen. Durch den universellen und nachsuchenden Zugang werden vom schulärztlichen Dienst dabei auch Familien und Kinder erreicht, die aus verschiedenen Gründen den Zugang zu einer hausärztlichen Versorgung verloren oder noch nicht etabliert haben.

\section{Schulärzte beobachten gesundheitliche Trends und fordern Massnahmen}

Dank der Nähe zur Schule eröffnet sich den Schulärztinnen und Schulärzten zudem ein breites Spektrum an Interventionen, welches die Massnahmen auf individueller Ebene wirksam ergänzt. So ist schulärztlicher Rat bei der Ausgestaltung der Verhältnisse in der 
Schule gefragt: Hygiene, Ergonomie, psychosoziale Fragen, Pausenplatzgestaltung, Ernährungs- und Bewegungsangebote oder die Situation der Umwelt sind nur einige Stichworte dazu. Der schulärztliche Einblick in die Situation ganzer Klassen- oder Schulkollektive gibt wichtige Hinweise auf problematische Trends und allenfalls bestehenden Handlungsbedarf. Kraft ihres schulärztlichen Amtes haben Schulärztinnen und Schulärzte die Legitimation, die verantwortlichen Schulleitungen, Schulbehörden oder politischen Entscheidungsträger auf bestehenden Handlungsbedarf hinzuweisen oder für die betroffenen Kinder und Jugendlichen notwendige Massnahmen einzufordern. Die Mitwirkung in interdisziplinären Schulgesundheitsteams oder an gesundheitsbezogenen Schüleroder Elternanlässen sind weitere Gelegenheiten, die Gesundheit der Schülerinnen und Schüler im Kollektiv zu fördern. Eine wichtige Rolle nehmen Schulärztinnen und Schulärzte zudem in der Bekämpfung von Infektionskrankheiten in der Schule sowie im Impfwesen ein.

Die schulärztliche Tätigkeit ist in den meisten Kantonen sowohl in der Gesundheits- als auch in der Bildungsgesetzgebung verankert. Meist sind die Rahmenbedingungen kantonal geregelt, und die Schulärztinnen und Schulärzte werden von den Schulgemeinden beauftragt und ernannt. Schulärztliche Dienste sind ein wichtiges Element des öffentlichen Gesundheitswesens in der Gemeinde. Das ist angesichts der lebenslang hohen Bedeutung einer gesunden Entwicklung und guten Bildung der Kinder gerechtfertigt.

\section{Schulärzte brauchen besondere Qualifikationen und Fachwissen}

Damit die schulärztliche Arbeit wirksam geleistet werden kann, braucht es spezifische Qualifikationen. Dazu gehören die kompetente Durchführung der Screening-Untersuchungen und die Einschätzung der schulrelevanten Gesundheits- und Entwicklungsaspekte gerade auch in der Adoleszenz. Darüber hinaus braucht es weiteres schulärztliches Fachwissen, so die gute Kenntnis der relevanten gesetzlichen Vorgaben und Verordnungen in den Bereichen Bildung und Gesundheit (z.B. zu besonderen schulischen Massnahmen, Nachteilsausgleich, Sonderschulung, oder Infektionsschutz) oder ein gutes Verständnis der Funktionsweise und Möglichkeiten der Schule und der schulnahen Dienste. Die vielfältigen obgenannten

\section{CAS Schulärztin/Schularzt}

Ab Herbst 2016 starten die Zürcher Hochschule für Angewandte Wissenschaften (ZHAW) und die von acht Schweizer Universitäten getragene Stiftung der Swiss School of Public Health (SSPH+) gemeinsam ein CAS Schulärztin/Schularzt, welches in der Schweiz erstmals eine umfassende Qualifizierungsmöglichkeit für Schulärztinnen und Schulärzte bietet. Mit Rücksicht auf die individuell unterschiedlichen Qualifizierungsbedürfnisse der schulärztlich tätigen Kolleginnen und Kollegen ist das CAS modular aufgebaut. Die Module und Kurse können auch einzeln im Rahmen der ärztlichen Fortbildungspflicht besucht werden. Drei Kurse sind inhärenter Bestandteil des postgraduierten SSPH+ Master of Public Health der Universitäten Basel, Bern und Zürich bzw. können darin angerechent werden. Das Weiterbildungsangebot unterstützt Schulärztinnen und Schulärzte mit der Stärkung ihrer interdisziplinären Qualifikationen und trägt damit zur klaren Profilierung der schulärztlichenTätigkeit bei. Der Einstieg ins CAS ist laufend möglich. Kursdaten sind im Kongresskalender oder auf folgender Webseite ersichtlich: https://weiterbildung.zhaw.ch/ de/gesundheit/programm/cas-schulaerztin-schularzt-school-healthprofessional.html

schulärztlichen Interventionsmöglichkeiten belegen zudem sehr deutlich, dass Schulärztinnen und Schulärzte überaus wichtige Aufgaben im Bereich Public Health wahrnehmen - Aktivitäten also, welche den Fokus nicht beim einzelnen Schulkind haben, sondern das «System Schule» und dessen gesamtes Potential in der Prävention und der Gesundheitsförderung mit berücksichtigen müssen. Das Konzept der «gesunden Schule» ist von grosser gesundheitspolitischer Tragweite. Es ist wichtig, dass der Schulärzteschaft in diesem interprofessionellen Handlungsfeld, zu dem viele an Universitäten und Fachhochschulen ausgebildete Berufsgruppen beitragen müssen, eine wichtige Rolle erhalten bleibt. Das fachlich sehr breite Betätigungsfeld bedingt den Erwerb entsprechender Qualifikationen. Da sie nicht alle Gegenstand der Ausbildung im Medizinstudium sind, gehören sie zu den Inhalten der Fort- und Weiterbildung. Für die Anerkennung und Wertschätzung der schulärztlichen Tätigkeit wäre es sehr zu begrüssen, wenn möglichst alle Kantone und Gemeinden freiwillige Fortbildungsmöglichkeiten ihrer Schulärztinnen und Schulärzte fördern und einen Teil der dafür anfallenden Kosten für sie übernehmen würden.

\section{Disclosure statement}

Julia Dratva und Annemarie Tschumper werden im CAS an je zwei Kurstagen als Dozentinnen involviert sein. Die Swiss School of Public Health (SSPH+) ist universitärer Partner des CAS Schulärztin/ Schularzt. 